\title{
FOTOINTERPRETAÇÃO COMPARATIVA DE FOTOGRAFIAS PANCROMÁTICAS COLORIDAS E INFRA- VERMELHAS COLORIDAS ATRAVÉS DA DRENAGEM *
}

\author{
Eng..$^{\circ}$ Agr. ${ }^{\circ}$ Gilberto J. Garcia *** \\ ENG. ${ }^{\circ}$ AGR. ${ }^{\circ}$ Delmar A. B. Marchetti ***
}

\section{RESUMO}

O presente trabalho teve por objetivo avaliar quantitativamente as informações obtidas por diferentes tipos defotografias.

Os estudos basearam-se em fotografias pancromáticas, coloridas e infravermelhas coloridas de uma área teste localizada no município de Campinas - SP.

A cobertura fotográfica denominada Missão 96 resultou da colaboração científica entre INPE - Instituto de Pesquisas Espaciais, NASA $\rightarrow$ National Aeronautics and Space Administration e IA.C - Instituto Agronômico do Estado de São Paulo.

A rede de drenagem foi o elemento de comparação tendo sido estudados dois parâmetros: número de segmentos de rios e comprimentos de rios.

A rede de drenagem foi estudada em monoscopia e em estereoscopia, onde as medições foram efetuadas em amostras circulares de $10 \mathrm{~km}^{2}$ (parcelas), sendo que o delineamento experimental foi o de parcelas inteiramente casualizadas.

Os dados obtidos foram utilizados para avaliar a eficiência de cada tipo de fotografia, para os parâmetros considerados. Para as condições estudadas, obteve-se as seguintes conclusões principais: a) a fotografia infravermelha forneceu maior número de informações comparativamente às fotografias pancromáticas e coloridas, as quais ofereceram resultados semelhantes; $b$ ) os resultados obtidos pela análise monoscópica da fotografia infravermelha foram semelhantes aos resultados obtidos pela análise estereoscópica da fotografia pancromática, o que pode facilitar bastante o processo fotointerpretativo.

* Enviado para publicação em 14/12/1976

* Trabalho apresentado no VI Congresso Nacional de Engenharia Agrícola 1976 - Centro Nacional de Engenharia Agrícola (Fazenda Ipanema - SP)

** Dept.o de Engenharia Rural - Faculdade de Ciências Médicas e Biológicas de Botucatu - UNESP

** * Dept.o de Engenharia Rural - Escola Superior de Agricultura "Luiz de Queiroz" - USP 


\section{INTRODUÇÃO}

A fotografia aérea desempenha um papel relevante no estudo dos recursos naturais de uma área. No caso específico de solos, o seu emprego é de extrema importância, tanto no que diz respeito à sua identificação, como na representação cartográfica das diferentes unidades pedológicas, simples e associadas.

Pela soma de informações que oferece, a rede de drenagem apresenta-se como um dos elementos de reconhecimnto mais importantes. A partir do estabelecimento de correlações entre terreno e fotografias aéreas, a análise dos elementos estudados possibilita a identificação dos tipos de solos de uma região. A utilização de fotografias coloridas e infravermelhas coloridas, com vantagens adicionais, aumentariam a importância dos dados. A presente pesquisa propõe-se a quantificar algumas destas vantagens adicionais, com vistas a futuros trabalhos.

O filme pancromático é sensível a todos os comprimentos de onda visíveis e, por ter uma faixa de sensibilidade semelhante à do olho humano, é considerado o melhor para usos gerais. Conforme cita DUTTON ${ }^{(5)}$, tal tipo de filme apresenta como principais vantagens: a) grande latitude de exposição; b) facilidade de manuseio e processamento; c) custo relativamente baixo; d) velocidade de exposição mais elevadas; e) menor susceptibilidade a fungos. Como desvantagens apresenta: a) as imagens são registradas somente em tons cinzas, fazendo com que objetos de mesma refletividade produzem imagens com mesma tonalidade; b) ausência de limite de cores, diminuindo a percepção. Segundo a COMPANHIA KODAK ${ }^{(6)}$, a maioria dos filmes pancromáticos têm sua sensibilidade, em relação ao espectro eletromagnético, entre 350 e $720 \mathrm{~m}_{\mu}$.

Quanto ao filme colorido, o tipo mais utilizado é o reversível, apresentando-se sensível a todas as cores do espectro. DUTTON ${ }^{(5)}$ comenta que as vantagens do filme colorido são: a) o uso de filmes reversíveis evita a necessidade de cópias, podendo as observações serem feitas diretamente nas transparências; b) grande contraste de cor e brilho, aumentando a interpretabilidade das fotografias aéreas; c) a cor aumenta a velocidade, confiança e precisão do processo fotointerpretativo; d) maior possibilidade de diferenciação de objetos, visto que o olho humano é capaz de distinguir 20.000 combinações diferentes entre cores, contra 200 tons diferentes de cinza do filme pancromático. Como desvantagens tem-se: a) custo mais elevado que o filme pancromático; b) latitude de exposição mais restrita; c) perda de saturação da cor durante o processamento e d) a qualidade da imagem diminui com o aumento da altitude. Conforme a COMPANHIA KODAK ${ }^{(6)}$ sua sensibilidade espectral está entre 400 e $780 \mathrm{~m}_{\mu}$.

Quanto ao filme infravermelho colorido, DUTTON ${ }^{(6)}$ relata que suas vantagens são: a) pode ser usado em dais em que a névoa e a fumaça 
impossibilitam o uso dos filmes pancromáticos e colorido; b) alto poder de resolução para delinear umidade em certos tipos de florestas; c) grande contraste entre objetos. Como desvantagens tem-se: a) custo relativamente alto; b) latitude de exposição menor que do filme pancromático; c) o filme virgem é sensivel a variações de temperatura; d) o filme virgem não mantém a tonalidade das cores com a idade. O manual da COMPANHIA KODAK ${ }^{(5)}$ estabelece que a sensibilidade desse filme em relação ao espectro eletromagnético situa-se entre 400 e $900 \mathrm{~m} \mu$. Ainda segundo a Companhia Kodak necessariamente im filtro para a luz azul (amarelo - Wratten n. ${ }^{\circ} 12$ ) deve ser usado.

Quanto ao padrão de drenagem, LUEDER ${ }^{(9)}$ assinala que sua evolução é em grande parte função da relação infiltração/deflúvio. Esta razão está por sua vez, intimamente relacionada com as características do solo, embora o clima, o relevo e a cobertura vegetal exerçam influência. Solos relativamente arenosos, devido a textura grosseira, favorecem a infiltração em detrimento do deflúvio, mostrando um padrão de drenagem pouco denso. Solos relativamente argilosos, oferecem maior resistência à infiltração, favorecendo o deflúvio e criando um padrão de drenagem mais denso.

Coube a HORTON ${ }^{(8)}$ a expressão "composição da rede de drenagem" para indicar onúmero e o comprimento de rios nas diferentes ordens, ressaltando o elevado grau de significação hidrológica da composição. $\mathrm{Na}$ notação da rede de drenagem em ordens, considerou os tributários menores e não ramificados como sendo de $1 .^{\mathrm{a}}$ rodem, atribuindo ao rio principal a ordem mais elevada. Estabeleceu ainda dois índices quantitativos: a) Densidade de Drenagem (Dd), como sendo o comprimento médio de segmentos de rios por unidade de área; b) Frequiência de Rios ( $\mathrm{Fr}$ ), definida como o número de segmentos de rios por unidade de área. Vários autores testaram e comprovaram as proposições de Horton, dentre os quais MAXWELL ${ }^{(10)}$, STRAHLER ${ }^{(16)}$ e FRANÇA $^{(7)}$.

Quanto à amostragem de áreas, RAY e FISCHER(14) fizeram estudos sobre litologia, relacionando comprimentos de rios e áreas circulares de $10 \mathrm{~km}^{2}$, obtendo resultados consistentes. FRANÇA ${ }^{(7)}$, utilizou tal tipo de amostragem no estudo de solos e obteve resultados altamente saisfatórios. SOUZA ${ }^{(15)}$, concluiu que a caracterização quantitativa da rede de drenagem pode ser efetuada com vantagens artavés do uso de amostras circulares, permitindo inclusive uma melhor amoseragem.

Vários estudos comparativos já foram realizados, utilizando-se dos filmes pancromático, colorido e infravermelho colorido. Segundo ANSON $^{(1)}$, o filme infravermelho colorido foi superior ao filme pancromático e ao colorido no estudo da rede de drenagem. Valentine et alii, citados por CARROL ${ }^{(3)}$, relatam que a fotografia infravermelha foi superior à pancromática no estudo de canais, comentando que provavelmente tal fato seria devido ao maior teor de umidade dos materiais superficiais. 


\section{MATERIAL E MÉTODOS}

\section{1 - Descrição da região estudada}

Os estudos basearam-se em fotografias pancromáticas, coloridas e infravermelhas coloridas de uma área teste localizada no município de Campinas - SP. Tal tipo de cobertura fotográfica, denominada Missão 96, resultou da colaboração científica entre INPE - Instituto de Pesquisas Espaciais, NASA - National Aeronautics and Space Administration e IAC - Instituto Agronômico do Estado de São Paulo. Esta região foi escolhida pela quantidade de dados disponíveis, tais como: tipo de solo, estações de plantio bem definidas e presença de vegetação natural.

A área teste está localizada entre as latitudes ( $\mathrm{Sul}$ ) de $22^{\circ} 35^{\prime}$ e $22^{\circ} 50^{\prime}$ e longitudes (Oeste) de $47^{\circ} 05^{\prime}$ e $47^{\circ} 20^{\prime}$.

CAMARGO $^{(2)}$ relata que o clima da região é do tipo tropical de altitude, com a temperatura do mês mais quente igual a $22,8^{\circ} \mathrm{C}$ e a do mês mais frio de $16,6^{\circ} \mathrm{C}$, sendo menor que $30 \mathrm{~mm}$ a precipitação do mês mais seco, de um total de $1.388 \mathrm{~mm}$ durante o ano.

\section{$2.2-$ Solos}

De acordo com a COMISSÃO DE SOLOS(4) a área apresenta na sua quase totalidade, solos da categoria Latossolo, especialmente do Latosolo Roxo e Latossolo Vermelho Escuro - Orto, conforme informações verbais da Seção de Pedologia do Instituto Agronômico do Estado de São Paulo.

O relevo da região é normal, suavemente ondulado, com declives mistos, médios a longos e irregulares, com declividades máximas de $8 \%$. A drenagem de tais solos é considerada boa, apresentando ainda perfis bem desenvolvidos, profundos com uma textura que varia de barrenta para argilosa.

\section{3 - Estereoscópio e instrumentos de medição e desenho}

Para observação estereoscópica dos pares fotográficos, foram utilizados dois tipos de estereoscópios: um de espelhos Wild ST4, e um de bolso marca Vasconcellos.

Foram empregados diversos instrumentos simples para as medições feitas e mmapas decalcados das fotografias aáreas. Entre eles estão: escala métrica, planímetro polar e curvímetro. 


\section{4 - Filmes e fotografias aéreas}

Os filmes utilizados no presente levantamento são produzidos comercialmente pela Companhia Eastman Kodak.

O filme pancromático utilizado foi o KODAK Plus X Aerographic Film 2402 (Estar base). É um filme preto e branco de granulação média, negativo, velocidade média, contraste médio, prestando-se para usos gerais em mapeamento e reconhecimento para altitudes médias a elevadas.

O filme colorido empregado no levantamento foi o KODAK EKTACHROME MS Aerographic Film 2448 (Estar base). E um filme de granulação fina, velocidade média, reversível, apresentando uma excelente definição e uma boa qualidade de imagem. É utilizado para mapeamento e reconhecimento para altitudes baixas a médias.

Quanto ao filme infravermelho colorido, foi utilizado o KODAK Aerochrome Infrated Film 2443 (Estar base). Com este tipo de filme, é necessário o uso de um filtro amarelo tal como o Wratten $n .^{\circ} 12$, para bloquear a radiação azul.

As fotografias aéreas disponíveis estão na escala 1:15.000 e 1:3.500, tendo sido utilizadas as primeiras.

\section{5 - Obtenção dos mapas básicos de drenagem}

Para cada tipo de fotografia foram demarcados os divisores de água e a rede de drenagem completa, com todos os canais de drenagem permanentes e temporários. A rede de drenagem foi traçada com 0 auxílio de pares estereoscópicos e em fotografias individuais.

Em estereoscopia utilizou-se de fotografias alternadas, segundo recomendação de RABBEN ${ }^{(13)}$, de modo que uma fotografia de cada par estereoscópio ficava livre de anotação, facilitando a análise. Foi feito finalmente um mapa geral da drenagem, através de triangulação radial, conforme descrito por NAMI e SLEPETYS(11).

Ao final dos trabalhos contava-se com três mapas básicos de drenagem em monoscopia e três em estereoscopia.

\section{$2.6-$ Amostragem}

No estudo da rede de drenagem, adotou-se a amostragem proposta por RAY e FISCHER ${ }^{(14)}$, para interpretação litológica e adaptada por FRANÇA $^{(7)}$ para solos. Assim, foram utilizadas amostras de $10 \mathrm{~km}^{2}$, com as necessárias repetições. 


\section{7 - Análise da rede de drenagem}

A drenagem no trabalho em questão é um parâmetro para o estudo comparativo dos três tipos de fotografias. Foram estudados os seguintes elementos da composição da rede de drenagem:

a) ordem da ramificação: os segmentos de rios ou canais de drenagem foram classificados com base na ordem de ramificação, segundo trabalho de STRAHLER ${ }^{(16)}$. Desse modo os canais de $1 .{ }^{\mathrm{a}}$ ordem vêm a ser as cabeceiras de erosão; $2{ }^{a}$ ordem, os canais resultantes da fusão dos primeiros; $3 .^{\mathrm{a}}$ ordem, os canais formados a partir da junção de canais de $2 .^{\text {a }}$ ordem.

b) número de segmentos de rios: computou-se os segmentos de rios em cada ordem e o número total na amostra.

c) comprimento de rios: foram medidos com o auxílio do curvímetro os comprimentos dos segmentos de rios de cada ordem e o comprimento total na amostra.

\section{8 - Delineamento experimental e análise estatística dos resultados}

Adotou-se o delineamento experimental de parcelas inteiramente casualizadas, constituído de três tratamentos com sete repetições. Os tratamentos referiram-se aos rês ipos de filmes, sendo $\mathrm{T}_{1}-$ Fotografia pancromática; $\mathrm{T}_{2}$ - Fotografia colorida e $\mathrm{T}_{3}-$ Fotografia infravermelha colorida. As repetições constituiram-se nas amostras circulares de $10 \mathrm{~km}^{2}$.

Os dados referentes à rede de drenagem e vegetação foram submetidos à análise de variância, e as médias dos tratamentos foram comparadas pelo teste de Tukey. GOMES $^{(12)}$.

Os processos estatísticos adotados são os expostos em PIMENTEL

\section{RESULTADOS E DISCUSSÃO}

A FIGURA 1 mostra duas amostras circulares da drenagem da região estudada e os QUADROS seguintes as medidas realizadas e os resultados obtidos.

Os números de segmentos de rios nas diversas ordens para os diferentes tratamentos em monoscopia e eh estereoscopia, podem ser vistos no QUADRO 1. 


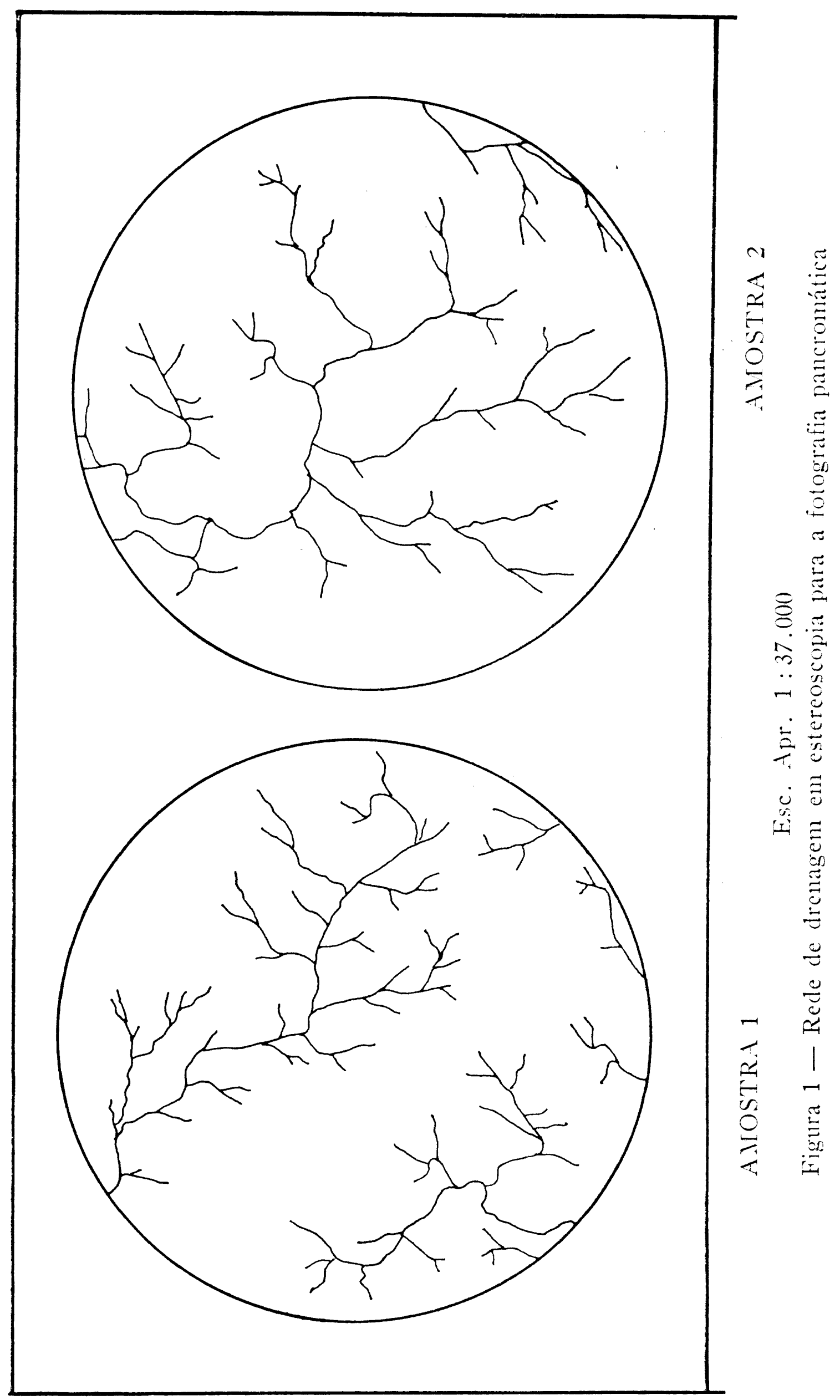


QUADRO 1 - Número de segmentos de rios nas diversas ordens em monoscopia e estereoscopia

\begin{tabular}{|c|c|c|c|c|c|c|c|c|c|c|}
\hline \multirow{2}{*}{$\begin{array}{c}\text { Especifi- } \\
\text { cações }\end{array}$} & \multirow{2}{*}{ Ordem } & \multirow{2}{*}{$\begin{array}{l}\text { Trata- } \\
\text { mento }\end{array}$} & 1 & 2 & 3 & 4 & 5 & 6 & 7 & \multirow{2}{*}{ Médias } \\
\hline & & & \multicolumn{7}{|c|}{ Repetições } & \\
\hline \multirow{12}{*}{ Mono } & \multirow{3}{*}{$1 .^{\mathrm{a}}$} & 1 & 47 & 38 & 30 & 59 & 52 & 33 & 50 & 44,14 \\
\hline & & 2 & 52 & 45 & 40 & 67 & 60 & 42 & 54 & 51,43 \\
\hline & & 3 & 68 & 63 & 57 & 82 & 72 & 55 & 69 & 66,67 \\
\hline & \multirow{3}{*}{$2 .^{a}$} & 1 & 15 & 12 & 10 & 18 & 12 & 11 & 17 & 13,57 \\
\hline & & 2 & 18 & 15 & 11 & 18 & 16 & 13 & 17 & 15,43 \\
\hline & & 3 & 24 & 17 & 17 & 26 & 20 & 19 & 24 & 21,00 \\
\hline & \multirow{3}{*}{$3 .^{a}$} & 1 & 3 & 3 & 4 & 3 & 1 & 2 & 2 & 2,57 \\
\hline & & 2 & 4 & 5 & 4 & 3 & 2 & 3 & 3 & 3,43 \\
\hline & & 3 & 5 & 6 & 5 & 6 & 5 & 5 & 6 & 5,43 \\
\hline & \multirow{3}{*}{$4 .^{\mathrm{a}}$} & 1 & 1 & 1 & 0 & 0 & 0 & 0 & 0 & 0,29 \\
\hline & & 2 & 1 & 1 & 0 & 0 & 0 & 1 & 0 & 0,43 \\
\hline & & 3 & 1 & 1 & 0 & 1 & 2 & 1 & 2 & 1,14 \\
\hline \multirow{12}{*}{ Estereo } & \multirow{3}{*}{$1 .^{\mathrm{a}}$} & 1 & 75 & 53 & 54 & 65 & 62 & 67 & 67 & 63,29 \\
\hline & & 2 & 77 & 60 & 56 & 65 & 62 & 71 & 73 & 66,29 \\
\hline & & 3 & 89 & 79 & 81 & 105 & 87 & 97 & 87 & 89,43 \\
\hline & \multirow{3}{*}{$2 .^{\mathrm{a}}$} & 1 & 24 & 16 & 17 & 22 & 16 & 18 & 21 & 19,14 \\
\hline & & 2 & 21 & 16 & 17 & 21 & 17 & 19 & 21 & 18,86 \\
\hline & & 3 & 27 & 20 & 22 & 30 & 27 & 25 & 24 & 25,00 \\
\hline & \multirow{3}{*}{$3 .^{\mathrm{a}}$} & 1 & 4 & 6 & 5 & 4 & 3 & 5 & 5 & 5.00 \\
\hline & & 2 & 3 & 6 & 5 & 4 & 3 & 5 & 4 & 4,29 \\
\hline & & 3 & & & & & & & & \\
\hline & \multirow{3}{*}{$4 .^{\mathrm{a}}$} & 1 & 1 & 1 & 1 & 1 & 1 & 1 & 1 & 1,00 \\
\hline & & 2 & 1 & 1 & 1 & 1 & 1 & 1 & 1 & 1,00 \\
\hline & & 3 & 1 & 1 & 1 & 1 & 1 & 1 & 2 & 1,14 \\
\hline
\end{tabular}


Os comprimentos de rios nas diversas ordens, para os diferentes tratamentos, em monoscopia e estereoscopia, são mostrados no QUADRO 2.

QUADRO 2 - Comprimento de rios nas diversas ordens em monoscopia e estereoscopia

\begin{tabular}{|c|c|c|c|c|c|c|c|c|c|c|}
\hline \multirow{2}{*}{$\begin{array}{c}\text { Especifi- } \\
\text { cações }\end{array}$} & \multirow{2}{*}{$\begin{array}{l}\text { Ordem } \\
\text { Trata- } \\
\text { mento }\end{array}$} & \multicolumn{8}{|c|}{ Repetições } & \multirow{2}{*}{ Médias } \\
\hline & & 1 & 2 & 3 & 4 & 5 & 6 & 7 & & \\
\hline \multirow{12}{*}{ Mono } & \multirow{3}{*}{$1 .^{a}$} & 1 & 7,6 & 6,8 & 5,9 & 8,7 & 8,3 & 6.4 & 8,3 & 7,42 \\
\hline & & 2 & 8,6 & 8,0 & 7,5 & 8,2 & 9,0 & 7,9 & 9,0 & 8,31 \\
\hline & & 3 & 13,1 & 13,1 & 13,3 & 10,1 & 10,6 & 9,4 & 12,3 & 11,70 \\
\hline & \multirow{3}{*}{$2 .^{a}$} & 1 & 5,1 & 6,8 & 6,0 & 6,0 & 4,0 & 5,6 & 7,1 & 5,80 \\
\hline & & 2 & 7,5 & 7,0 & 6,0 & 6,0 & 5,6 & 6,0 & 7,0 & 6,44 \\
\hline & & 3 & 8,3 & 9,0 & 8,3 & 7,5 & 6,7 & 7,0 & 8,3 & 7,87 \\
\hline & \multirow{3}{*}{$3 .^{a}$} & 1 & 4,0 & 1,9 & 2,3 & 4,1 & 3,8 & 3,8 & 3,4 & 3,33 \\
\hline & & 2 & 4,5 & 3,0 & 3,0 & 4,8 & 3,8 & 4,1 & 4,1 & 3,90 \\
\hline & & 3 & 3,8 & 3,4 & 3,8 & 4,5 & 3,0 & 4,0 & 4,9 & 3,91 \\
\hline & \multirow{3}{*}{$4 .^{a}$} & 1 & 0,4 & 1,9 & 0 & 0 & 0 & 0 & 0 & 0,33 \\
\hline & & 2 & 0,4 & 2,3 & 0 & 0 & 0 & 0,8 & 0 & 0,50 \\
\hline & & 3 & 2,0 & 2,3 & 3,8 & 1,1 & 2,2 & 2,2 & 1,3 & 2,13 \\
\hline \multirow{12}{*}{ Estereo } & \multirow{3}{*}{$1 .{ }^{\mathrm{a}}$} & 1 & 11,2 & 8,6 & 11,3 & 15,3 & 13,6 & 15,5 & 14,6 & 12,87 \\
\hline & & 2 & 15,1 & 13,1 & 13,0 & 16,2 & 11,8 & 15,2 & 11,1 & 13,64 \\
\hline & & 3 & 18,8 & 18,4 & 17,8 & 20,8 & 15,5 & 18,3 & 21,0 & 18,66 \\
\hline & \multirow{3}{*}{$2 .^{\mathrm{a}}$} & 1 & 7,3 & 6,4 & 6,2 & 6,4 & 10,2 & 7,5 & 8,1 & 7,44 \\
\hline & & 2 & 8,0 & 8,7 & 6,5 & 6,0 & 6,0 & 9,3 & 7,7 & 7,46 \\
\hline & & 3 & 9,8 & 8,5 & 9,3 & 9,0 & 7,3 & 8,1 & 10,5 & 8,93 \\
\hline & \multirow{3}{*}{$3 .^{\mathrm{a}}$} & 1 & 4,1 & 5,1 & 4,0 & 3,8 & 2,3 & 4,5 & 4,1 & 3,99 \\
\hline & & 2 & 4,9 & 3,8 & 4,0 & 3,8 & 2,3 & 4,1 & 3,8 & 3,81 \\
\hline & & 3 & 4,1 & 4,9 & 5,6 & 4,9 & 3,0 & 5,3 & 4,5 & 4,61 \\
\hline & \multirow{3}{*}{$4 .^{\mathrm{a}}$} & 1 & 2,3 & 1,5 & 0,8 & 1,1 & 0,8 & 0,7 & 0,8 & 1,14 \\
\hline & & 2 & 0,6 & 1,5 & 0,6 & 1,1 & 1,9 & 0,8 & 0,8 & 1,04 \\
\hline & & 3 & 1,9 & 0,8 & 0,4 & 0,6 & 2,3 & 2,7 & 1,5 & 1,46 \\
\hline
\end{tabular}


Quanto ao número de segmentos de rios, o QUADRO 3 mostra que a análise de variância dos dados obtidos para os três tratamentos revelou que os resultados foram significativos ao nível de $1 \%$ de probabilidade até $3 .^{a}$ ordem, não sendo significativos os resultados de 4 . $^{\text {a }}$ ordem. A comparação de médias feitas pelo teste de Tukey, tanto para os dados monoscópicos como estereoscópicos, mostrou que os tratamentos 1 e 2 são estatisticamente semelhantes, diferindo significativamente do tratamento 3. Pode-se perceber ainda pelo mesmo quadro, que o tratamento 3 em monoscopia é estatisticamente semelhante ao tratamento ao tratamento $1 \mathrm{em}$ estereoscopia. O fato do número de segmentos de rios, para a fotografia infravermelha colorida em monoscopia, ser semelhante ao número de segmentos de rios para a fotografia pancromática em estereoscopia, revela-se interessante. Desse modo, havendo fotografia infravermelha colorida de uma região, a drenagem poderia ser estudada em monoscopia, faciiltando os trabalhos e obtendo valores comparáveis aos já obtidos através da análise estereoscópica de fotografias pancromáticas de outras regiões. $O$ efeito do tipo de fotografia revelou-se maior em monoscopia, facilitando os trabalhos e obtendo valores comparáveis aos já obtidos através da análise estereoscópica de fotografias revelou-se maior em monoscopia, e isto se deve à maior sensibilidade do filme infravermelho à umidade do solo. Embora os canais de $1 .^{a}$ ordem sejam, em sua maioria, cursos temporários de água, é de se esperar que o solo apresente, dentro de certos limites, teores maiores de umidade, sendo possível a localização desses caanis. Em estereoscopia o efeito do tipo de fotografia apresenta-se um pouco menor. O fato dos canais de primeira ordem já apresentarem um certo sulcamento possibilita a sua visualização em estereoscopia, minimizando o efeito do tipo de fotografia. Porcentualmente a fotografia infravermelha colorida registrou maior número de segmentos de rios nas diversas ordens que a fotografia pancromática. Desse modo obteve-se $33,7 \% ; 35,4 \%$ e $52,7 \%$ para os valores de $1 .^{\mathrm{a}}, 2 .^{\mathrm{a}}$ e $3 .^{\mathrm{a}}$ ordem respectivamente. Em estereoscopia obteve-se $29,2 \% ; 23,4 \%$ e $14,7 \%$ para os canais de $1 .^{a}, 2 .^{a}$ e 3. ${ }^{a}$ ordem respectivamente. Pelos dados acima pode-se perceber que o número de segmentos de rios em nonoscopia, cresceu a medida que aumentou a ordem de ramificação, enquanto que em estereoscopia ocorreu o inverso. Isto pode ser devido ao fato de que em monoscopia, somente os canais perfeitamente visíveis são demarcados. Desse modo dificilmente os canais de ordens superiores deixariam de ser diagnosticados, aumentando em termos porcentuais as suas participações.

Quanto ao comprimento de rios, o QUADRO 4, mostra que a análise de variância dos dados obtidos para os três tratamentos revelou que os resultados foram significativos ao nível de $1 \%$ de probabilidade até $2 .^{a}$ ordem, não tendo significância os valores de $3 .^{a}$ e $4 .^{a}$ ordens. A comparação de médias feita pelo teste de Tukey, tanto para os dados monoscópicos como estereoscópicos, mostrou que os tratamentos 1 e 2 são estatisticamente semelhantes, e diferem significativamente do tratamento 3. Neste caso também, o comprimento de rios para a fotografia infravermelha colorida em monoscopia foi semelhante ao comprimento 
QUADRO 3 - Análise de variância e teste de Tukey para os Números de segmentos de rios nas diversas ordens.

\begin{tabular}{l|cccc}
\hline \multirow{2}{*}{ Tratamentos } & \multicolumn{4}{|c}{ Ordem de Ramificação-Médias } \\
\cline { 2 - 5 } & $1 .^{\mathrm{a}}$ & $2 .^{\mathbf{a}}$ & $3 .^{\mathbf{a}}$ & $4^{\mathbf{a}}$ \\
\hline \hline 1 Mono & 44,14 & 13,57 & 2,57 & 0,29 \\
2 Mono & 51,43 & 15,43 & 3,43 & 0,43 \\
3 Mono & 66,57 & 21,00 & 5,43 & 1,14 \\
\hline 1 Estereo & 63,29 & 19,14 & 5,00 & 1,00 \\
2 Estereo & 66,29 & 18,86 & 4,29 & 1,00 \\
3 Estereo & 89,43 & 25,00 & 5,86 & 1,14 \\
\hline F. Trat. & $20,54 * *$ & $8,79 * *$ & $12,14 * *$ & $\mathrm{NS}$ \\
DMS 5\% & & & & \\
\hline
\end{tabular}

(Tukey)

Valores significativos ao nível de $1 \%$ de probabilidade

QUADRO 4 - Análise de variância de teste de Tukey para os Comprimentos de rios nas diversas ordens.

\begin{tabular}{lrl|ll}
\hline \hline 1 Mono & 7,42 & 5,80 & 3,33 & 0,33 \\
2 Mono & 8,31 & 6,44 & 3,90 & 0,50 \\
3 Mono & 11,70 & 7,87 & 3,91 & 2,13 \\
\hline 1 Estereo & 12,81 & 7,44 & 3,99 & 1,14 \\
2 Estereo & 13,64 & 7,46 & 3,81 & 1,04 \\
3 Estereo & 18,66 & 8,93 & 4,61 & 1,46 \\
\hline F. Trat. & $39,11 * *$ & $7,02 * *$ & $\mathrm{~N} \mathrm{~S}$ & $\mathrm{~N} \mathrm{~S}$ \\
\hline DMS 5\% & 2,77 & 1,75 & & \\
\hline
\end{tabular}

(Tukey)

Valores significativos ao nível de $1 \%$ de probabilidade 
de rios para a fotografia pancromática em estereoscopia, o que poderia facilitar o estudo da drenagem de uma região. Para o comprimento de rios, foram também os canais de primeira ordem que apresentaram as maiores variações, segundo o tipo de fotografia. Tomando-se como referência a fotografia pancromática, estas variações referiram-se a prolongamentos de canais já marcados e por novos canais encontrados com o auxílio da fotografia infravermelha. Pelo QUADRO 4 pode-se perceber ainda que o exame estereoscópico da fotografia infravermelha colorida forneceu duas vezes e meia mais informações em relação ao exame monoscópico da fotografia pancromática, para canais de $1 .^{a}$ ordem.

\section{CONCLUSÕES}

Para a região estudada os resultados permitem as seguintes conclusões principais:

a) no estudo da drenagem, para todos os parâmetros considerados, a fotografia infravermelha colorida forneceu maior número de informações, comparativamente à fotografia pancromática e colorida, as quais ofereceram resultados semelhantes.

b) os resultados obtidos através da análise monoscópica da fotografia infravermelha, foram semelhantes aos resultados obtidos pelo estudo estereoscópico da fotografia pancromática, o que pode facilitar o processo fotointerpretativo.

\section{SUMMARY \\ COMPARATIVE PHOTOINTERPRETATION OF PANCHROMATIC, COLOR AND COLOR INFRARED PHOTOS}

The present work had as its main aim a quantitative evaluation of information obtained through different types of photographs.

The studies were based on panchromatic, color and color infrared photographs of a test area located in the municipality of Campinas - SP.

The photographic flight was carried on by means of a scientific cooperation of INPE - Instituto de Pesquisas Espaciais, NASA - National Aeronautics and Space Administration and IAC - Instituto Agronômico do Estado de São Paulo and was known Mission 96.

The drainage was the parameter analysed, and were measured the quantity and lenght of channels.

The drainage network was studied monoscopically and stereoscopically, and the measurements were made in circular samples $10 \mathrm{~km}^{2}$, randomily orientated.

The obtained data were used to evaluate the efficiency of every of photograph, for the studied variables. 
On the basis of the present study, the quantitative analysis led to the following main conclusions: a) Color infrared photography was the best in the study of the drainage network quantitative characteristics, comparatively to panchromatic and color photographs; b) The results obtained in studying drainage through the monoscopic study of infrared color photography were similar to the results of stereoscopic analysis of the panchromatic photography, wich would present a better readiness in the photointerpretation process.

\section{LITERATURA CITADA}

ANSON, A. 1966 - Color Photo Comparison. Photogram. Engng., 32 :286-297.

CAMARGO, A.P. de.' 1960 - Balanço Hídrico no Estado de São Paulo. Campinas, Instituto Agronômico, 1960. 20 p. (Boletim n. $\left.{ }^{\circ} 116\right)$.

CARROL, D.M. 1973 - Remote Sensing Techniques and Their Applications Science. I - Photographic Sensors. Soil and Fertilizers, $36: 259-266$.

COMISSÃO DE SOLOS DO C.N.E.P.A. 1964 - Levantamento de Reconhecimento dos Solos do Estado de São Paulo. Rio de Janeiro, Centro Nacional de Ensino e Pesquisas Agronômicas. $634 \mathrm{p}$.

DUTTON, J.A. 1967 - Comparative Photointerpretation from Panchromatic, Color and Color IR Photography, Ahio. 181 p. (Tese M.S. - Ohio State University).

EASTMAN KODAK COMPANY. 1972 - Applied Infrated Photography. N. York, 88 p. (Kodak Technical Publication. M-28).

FRANÇA, G.V. de. 1968 - Interpretação Fotográfica de Bacias e de Redes de Drenagem Aplicada a Solos da Região de Piracicaba. Piracicaba. 151 p. (Tese Doutoramento ESALQ).

HORTON, R.E. 1945 - Erosinal Development of Streams and Their Drainage Basins: Hidrophysical Approach to Quantitative Morfology. Bull. Geol. Soc. Am., 56:275-370.

LUEDER, D.R. 1969 - Aerial Photographic Interpretation: Principles and Applications. N. York, McGraw-Hill. 162 p.

MAXWELL, J.C. $1955 \rightarrow$ The Bifurcation Ratio in Horton's Law Stream Numbers (Resumo). Trans. Am. Geogh. Un., $36: 520$.

NAMI, A.J. e SLEPETYS, Z. 1953-1954 - Mosaicos Aerofotográficos. Engenharia Fotogramétrica. (2-6).

PIMENTEL GOMES, F. 1970 - Curso de Estatística Experimental. São Paulo, Livraria Nobel. $430 \mathrm{p}$.

RABBEN, E.L. 1960 - Fundamentals of Photo Interpretation. In: American Society of Photogrametry. Manual of Photographic Interpretation. Virginia. p. 99-186.

RAY, R.G. \& FISHER, W.A. 1960 - Quantitative Photography. A Geologic Research Tool. Photogram. Engng., $26: 143-150$.

SOUZA, M.L.P. 1975 - Fotointerpretação das Redes de Drenagem de Três Solos com Horizonte B Latossoólico Ocorrentes no Município de Ponta Grossa-PR. Piracicaba. 135 p. (Diss. Mestrado - ESALQ).

STRAHLER, A.N. 1957 - Quantitative Analysis of Watershed Geomorphology. Trans. Am. Geogh. Un., $38: 913-920$. 
